\title{
A Constitutive Model Incorporating Mixed Soil Slurry for Interface
}

\author{
Kai Peng ${ }^{1, *}$, Guohui Wang ${ }^{1}$, Yaolai Liu ${ }^{1}$, Xiaoliang Wang ${ }^{1}$, Dong Liu ${ }^{1}$,Liangliang Ma ${ }^{1}$ and Zhijun Bai ${ }^{1}$ \\ ${ }^{1}$ Powerchina Zhongnan Engineering Corporation Limited, Changsha, Hunan Province, 410014, China
}

\begin{abstract}
In projects, mixed soil slurry between cut-off wall and coarse-grained soil always exists. It may influence on or change mechanical properties of interface between coarse-grained soil and structure. The mechanical behaviors of the interface between coarse-grained soil and concrete were investigated by simple shear tests under mixed soil slurry. The significant dilatancy and stress-strain softening can be achieved through the results, which also indicate that the cement content play an important role in the shear strength of the interface. The peak strength and the position when the dilatancy occurs are related to both normal stress and cement content. An elasto-plastic constitutive model for interface considering mixed soil slurry was formulated in the framework of generalized potential theory. The entire model parameters can be identified by experimental tests. Finally, the predictions of the model have been compared with experimental results, and results show the model is reasonable and practical.
\end{abstract}

\section{Introduction}

Paster [1] developed a constitutive modeling which used loading direction vector and plastic flow direction vector instead of yield and plastic potential function based on the generalized theory of plastic. G.H.Yang [2] advised a new method to build soil constitutive model in mathematic way and presented a generalized potential theory which is easy to apply in building model.

Z.J.Zhang et al [3] analyzed influence on coarsegrained soil-concrete interface due to variation of slurry thickness and water content. M.Z.Zhang [4] analyzed the influence of mudcake on shaft friction.

In projects, due to construction technology, mixed soil slurry between cut-off wall and coarse-grained soil always exists, which may influence on or change mechanical properties of coarse-grained soil-structure, and then the load sharing ratio between the soil and concrete will change. Although many experimental and theoretical studies were performed to investigate behavior of interface, few of them were on slurry.

Therefore, the properties of the interface under mixed soil slurry were studied. An elasto-plastic constitutive model for thin layer element was presented and combined with results of test; The calculated values are all obtained in good agreement with experimental data by above models.

\section{Test results and analysis}

Simple shear apparatus was shown in Fig. 1(a). Thickness and inner diameter of each stacked ring were $20 \mathrm{~mm}$ and $300 \mathrm{~mm}$ respectively. The total effective height of sample was $120 \mathrm{~mm}$. In test, each stacked ring was set with shear displacement sensor and normal pressure $\left(\sigma_{n}\right)$ was given, shear displacement $(\delta)$ under different shear stress $(\tau)$ was measured and tangential strain $(\gamma)$ within thickness $(t)$ was obtained:

$$
\tau=T / A, \sigma_{n}=P / A, \gamma=\delta / t
$$

where $P, T$ refer to the vertical and horizontal load respectively, $A, t$ refer to area and thickness of the interface, as shown in Fig. 1(b).

The maximum particle diameter of coarse-grained soil was $20 \mathrm{~mm}$. Experimental dry density was $2.13 \mathrm{~g} / \mathrm{cm}^{3}$ and relative density was 0.9 . The density and water content of the clay were $1.64 \mathrm{~g} / \mathrm{cm}^{3}$ and $30 \%$ respectively. The liquid limit and plastic Limit of the clay were $34 \%$ and $20 \%$ respectively and specific gravity was 2.75 .

The cement content in the mixed soil slurry were $5 \%, 10 \%$ and $20 \%$ respectively. The water cement ratio of the cement slurry was $1.5: 0.5$. The interface tests were conducted till mixed soil slurry was cured $28 \mathrm{~d}$.

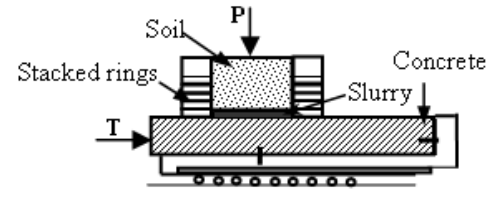

(a) Schematic diagram of experimental equipment

${ }^{*}$ Corresponding author's e-mail: 02509@msdi.cn 


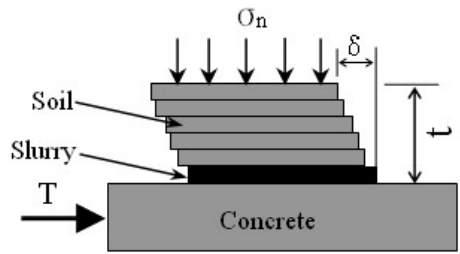

(b) Force of interface sample

Figure 1. Schematic diagram of simple shear test

The surface of the concrete was coarse. In view of $20 \mathrm{~mm}$ thickness of single stacked ring, the thickness of mixed soil slurry was made $20 \mathrm{~mm}$ so as to better reflect the deformation of the interface during the shear process.. The normal stresses were $200 \mathrm{kPa}, 500 \mathrm{kPa}, 1200 \mathrm{kPa}$ and $2000 \mathrm{kPa}$ respectively and the shear rate was $0.3 \mathrm{~mm} / \mathrm{min}$.

The relation curves between shear stress $(\tau)$ and tangential strain $(\gamma)$ were shown in Fig.2(a)- Fig.2(c) under different cement content. According to Fig.2 (a) Fig.2(c), stress-strain softening is obvious, and under the same normal stress, the more the cement content is, the larger the shear stress is. In addition, peak strength and its position increase with cement content and normal stress.

Fig.2(d)-Fig.2(f) was the relation curves between normal strain $\left(\varepsilon_{n}\right)$ and tangential strain $(\gamma)$. There is significant shear dilatancy, especially under small normal stress. In this paper, shear contraction was taken the positive value and shear dilatancy was taken the negative value.

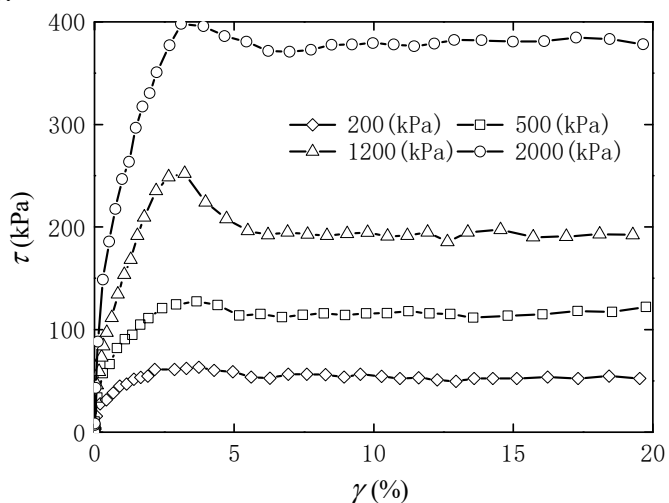

(a) Cement content (5\%)

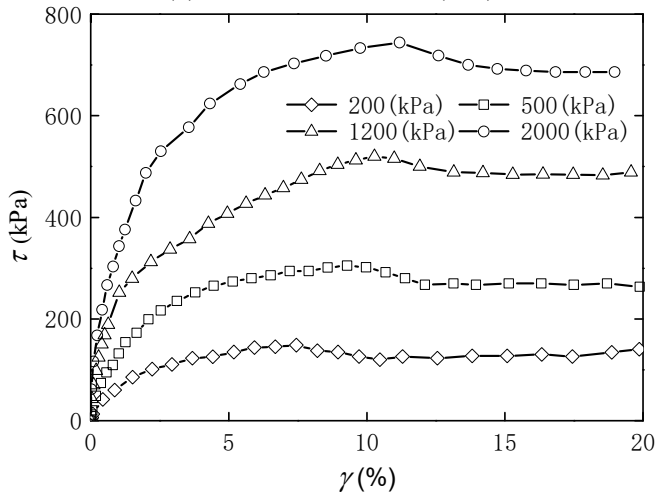

(b) Cement content (10\%)

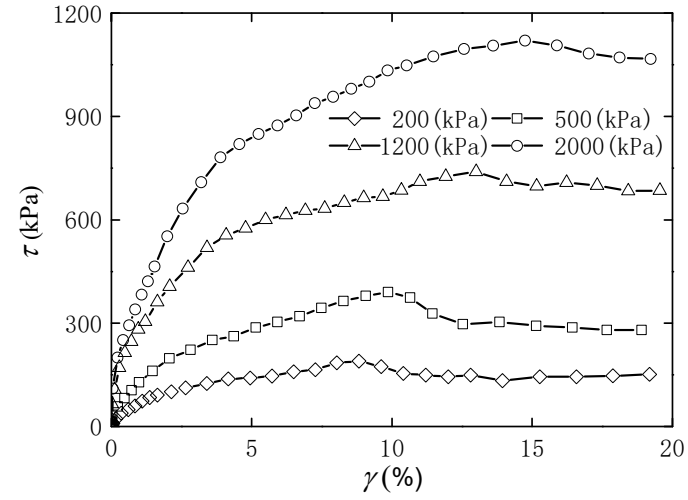

(c) Cement content $(20 \%)$

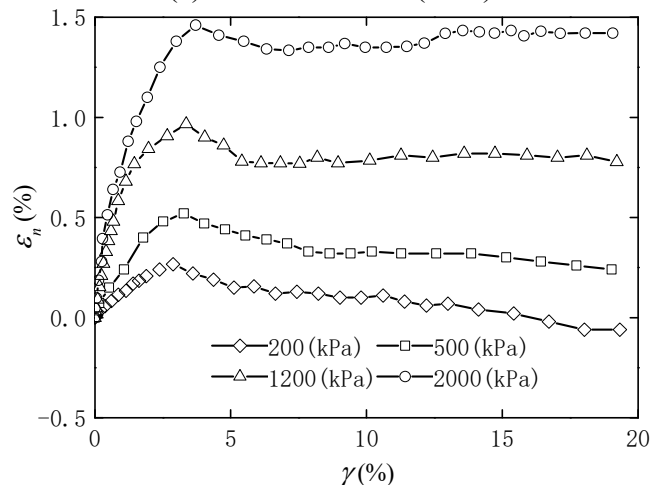

(d) Cement content $(5 \%)$

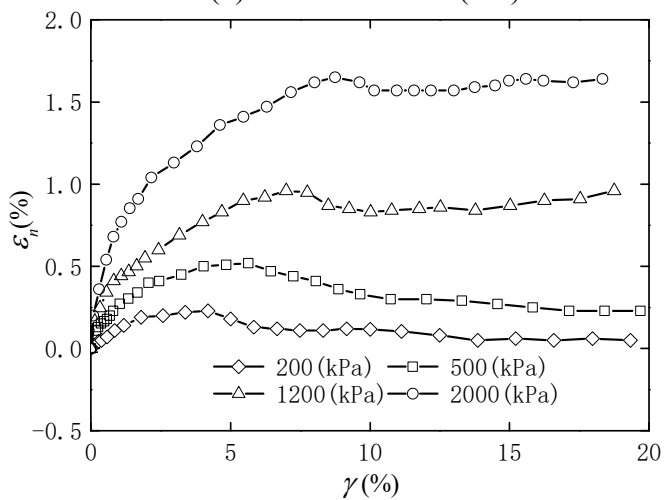

(e) Cement content $(10 \%)$

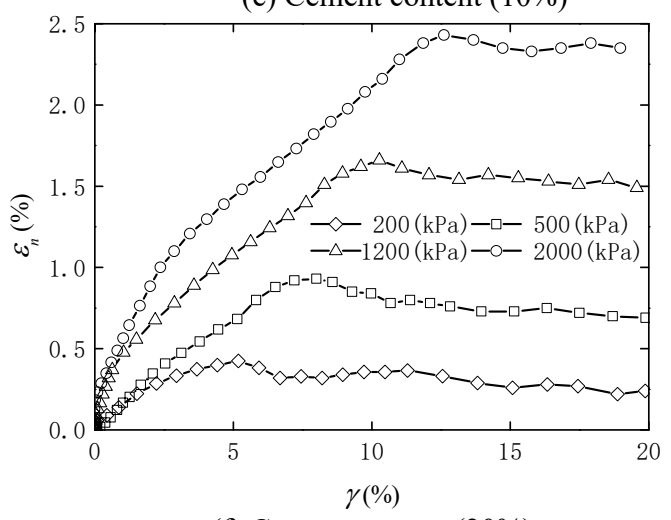

(f) Cement content $(20 \%)$

Figure 2. Curves of both $\tau \sim \gamma$ and $\varepsilon_{n} \sim \gamma$ under different cement content 


\section{An elasto-plastic constitutive model for interface}

\subsection{Main equations of elasto-plastic constitutive Model}

In shear test, a two-dimensional relation between stress and strain can be obtained, aka, relation between $\left(\sigma_{n}, \tau\right)$ and $\left(\varepsilon_{n}, \gamma\right)$. In stress space, its2-D vectors are $\{\boldsymbol{\varepsilon}\}=\left\{\boldsymbol{\varepsilon}_{\mathrm{n}}, \boldsymbol{\gamma}\right\}$ and $\quad\{\boldsymbol{\sigma}\}=\left\{\boldsymbol{\sigma}_{\mathrm{n}}, \boldsymbol{\tau}\right\} \quad$ respectively. Therefore, according to the generalized potential theory and elasto-plastic theory [5], the mathematical expressions of relation between total stress and strain are:

$$
\left.\begin{array}{l}
\mathrm{d} \varepsilon_{n}=\left(A+\frac{1}{K_{e}}\right) \mathrm{d} \sigma_{n}+B \mathrm{~d} \tau \\
\mathrm{d} \gamma=C \mathrm{~d} \sigma_{n}+\left(D+\frac{1}{G_{e}}\right) \mathrm{d} \tau
\end{array}\right\}
$$

Where $G_{e}$ refers to tangential elastic modulus. $K_{e}$ is normal elastic modulus, which was determined by simple compression curves and rebound curves. Further, $G_{e}$ was determined when Poisson ratio is assumed 0.3.

The coefficient matrix was composed of $A, B, C, D$ in the model, which can be obtained from the shear test. $A$ is coefficient reflecting compression due to normal stress, $B$ is coupled coefficient, which reflects influence of shear stress on normal strain, $D$ reflects shear properties. Besides, generally, simple or direct shear test does not consider the shear deformation caused by normal stress, therefore, $C=0$.All the coefficients were obtained if the plastic state equation was determined.

\subsection{Model parameters of the interface}

Under mixed soil slurry, the mechanical response of the interface was pictured as Fig.2. The plastic state equation was obtained by fitting the test results with piecewise function which was composed of hyperbolic function and resembling normal function. Eventually, parameters of the model were determined.

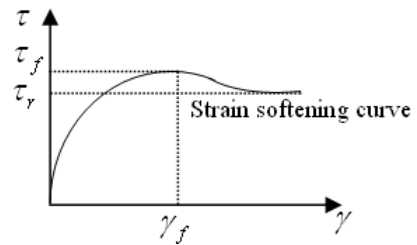

(a) Schematic diagram of $\tau \sim \gamma$

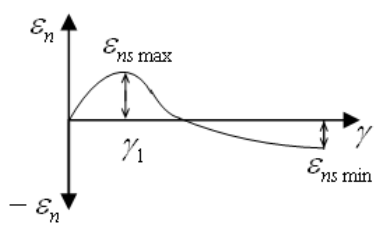

(b) Schematic diagram of $\varepsilon_{n} \sim \gamma$

Figure 3. Mechanical response of interface under mixed soil slurry.

\section{Verification and Discussions}

The simple experiment between gravel and concrete under the another mixed soil slurry (bentonite mixed with cement content $5 \%$ ) was conducted. The validity of the new model was verified by comparing the test data and calculated values with the new model on the space of both shear stress and normal strain. Fig.3gave the comparing results both experimental data and calculated values.

In Fig.3(a), the dilatancy is obvious when the normal tress is low, on the other hand, the stress-strain softening under large normal stress. And the values calculated by new model basically match the experimental data, the error generally within $5 \%$, maximum error is less than $10.5 \%$. Concerning other aspects of normal strain, as shown in Fig.3(b), the calculated values are also in agreement with the measured values, their deviation is within $12.7 \%$,

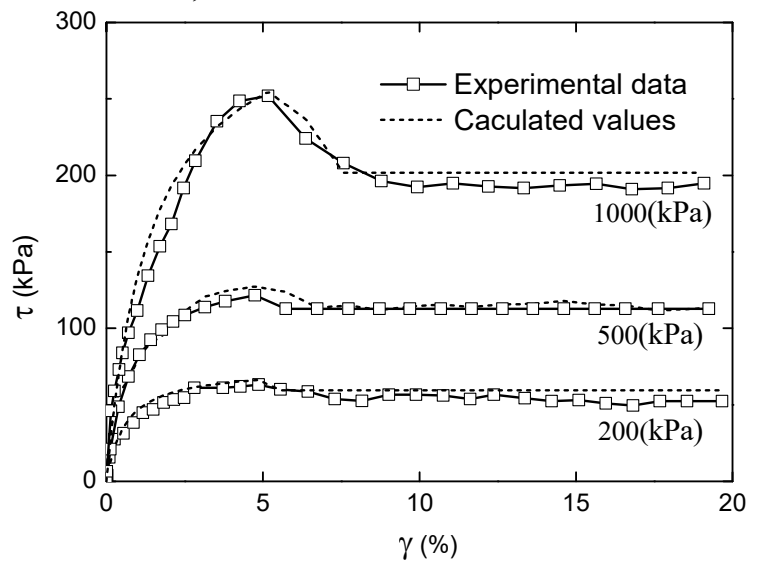

(a) $\tau-\gamma$ curves of test and fitting value

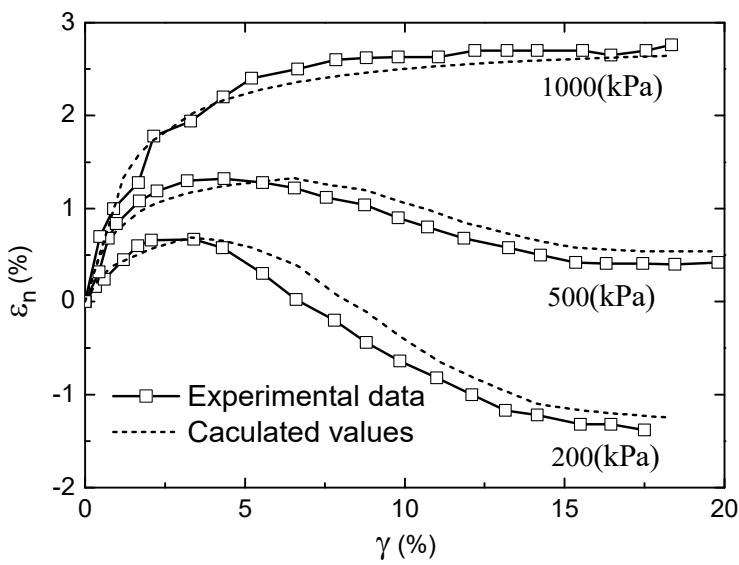

(b) $\varepsilon_{n}-\gamma$ curves of test and fitting value

Figure 4. Comparison between the test results and model value

\section{Conclusions}

(1) The interface under mixed soil slurry with different cement content was studied. The results reveal that the content of cement plays an important influence on the shear strength, however, the response of the interface such as the relationship between tangential strain and shear stress, as well as the relationship between tangential strain and normal strain, is not influenced by the cement content. 
(2)The relation curve between shear stress and tangential strain appears stress-strain softening under mixed soil slurry and dilatancy is apparent. The position of the peak strength is related to both the normal stress and cement content. In addition, shear dilatancy occurs before the peak strength.

(3)The 2-D constitutive model of the interface is presented based on the generalized potential theory. Plastic state equations are determined by fitting hyperbolic function and resembling normal function. Finally, model parameters are determined based on simple shear tests.

(4) The calculated value by new model is closed to experimental data, which verifies reasonability and applicability.

(5) The thickness of the mudcake, the content of cement and the saturation degree of the mixed soil slurry all have important effects on the mechanical properties of the contact surface, which need to be further studied.

\section{References}

1. M.Paster, O.C.Zienkiewicz and A.H.C.Chan :Generalized Plasticity and the Modeling of Soil Behavior.Int.J.Numer.and Anal.methods in geomechanics. 14 :151-190(1990)

2. G.h.Yang, Proceedings of Second International Conference on Soft Soil Engineering, edtied by Z. J. Shen, Hohai University Press, Nanjing, in press(1996)

3. Z.J.Zhang, X.B. Rao and Z.J.Wang.Experimental Study on Influence of Slurry Thickness on Mechanical Behavior of Interface between Gravel and Concrete. Rock and Soil Mechanics. 29(9): 2433 -2438 (2008)

4. M.Z.Zhang, G.X.Zhang and Q.Y.Wu. Studies on Characteristics of Mudcake and Soil between Bored Plies. Chinese Journal of Geotechnical Engineering. 28(6): 695-699 (2006)

5. A.Z.Zhou, T.H.Lu.Elasto-plastic Constitutive Model of Soil-structure Interface in Consideration of Strain Softening and Dilation.Acta Mechanics Solida Sinica. 22(2):171-179 (2009) 